\title{
Experimental observation of structural phase transition in CsBr clusters
}

\author{
L. Hautala, ${ }^{1, *}$ K. Jänkälä, ${ }^{1}$ T. Löytynoja, ${ }^{1}$ M.-H. Mikkelä, ${ }^{2}$ N. Prisle,${ }^{1}$ M. Tchaplyguine,${ }^{2}$ and M. Huttula ${ }^{1}$ \\ ${ }^{1}$ Nano and Molecular Systems research unit, University of Oulu, P.O. Box 3000, 90014 University of Oulu, Finland \\ ${ }^{2}$ MAX IV Laboratory, Lund University, Box 118, 22100 Lund, Sweden
}

(Received 8 September 2016; revised manuscript received 9 December 2016; published 3 January 2017)

\begin{abstract}
Formation and growth of $\mathrm{CsBr}$ clusters embedded in unsupported Ar clusters was studied using synchrotron radiation photoelectron spectroscopy. The development of the core-level electronic structure for cluster sizes between a few and a few hundred atoms contained information about the local coordination of the constituent particles. The experimental results indicate that a gradual structural phase transition from $\mathrm{NaCl}$ structure to $\mathrm{CsCl}$ structure for $\mathrm{CsBr}$ clusters takes place at around 160 atoms per cluster.
\end{abstract}

DOI: 10.1103/PhysRevB.95.045402

\section{INTRODUCTION}

Aerosol particles play crucial roles in atmospheric chemistry. Sea spray aerosols are injected into the air from various processes and comprise both inorganic sea salt and organic molecules [1]. The structure and chemical properties can play important roles for aerosol-water interactions. Varying water uptake on aerosol particles mixed with different marine halides [2] may be linked to different propensities of the halides for the immediate aqueous interface. Although sea salt aerosols are generally mixtures of different inorganic salts, pure component structural information is a crucial first step to assess the water interaction potential of different sea salt components and in particular investigate the possibly much enhanced role of the less abundant components. The ability to study neutral salt clusters could have a highly significant impact on our current understanding of the role of charge in atmospheric nucleation processes.

Alkali halide clusters have been studied in many forms over the last several decades. Many have involved characterization of cluster structure via their sequence of exceptionally stable cluster sizes observed in mass spectroscopic data [3-5]. Other ways of obtaining information about structural properties have included ion mobility measurements [6] and electron diffraction [7] performed on trapped cluster ions. Common for all of the techniques mentioned is that the cluster needs to be charged. Photoelectron spectroscopy has been applied to neutral alkali halide clusters [8-10] as the technique in itself does not rely on any specific charge state, unless size selection of clusters is of interest prior to photoemission. Recent experiments have greatly benefited from the increased photon flux and energy range of synchrotron radiation light sources. This has made it possible to study the electronic core levels of unsupported free alkali halide clusters [8-10] with sufficient signal intensity.

The study of core levels on alkali halide clusters provides information about the local chemical environment in which the atoms (or in this case, ions) are located. Structural properties like bond length and coordination as well as atomic properties such as polarizability constitute the chemical environment thus affecting the core-level energies. If the core-level energies are changed by these local effects to an extent that differ-

\footnotetext{
*1auri.hautala@oulu.fi
}

ent coordination sites and their relative abundance can be inferred experimentally, the global cluster geometry can be reconstructed by comparing these results to statistical models of known cluster structures.

$\mathrm{CsBr}$ is one of the alkali halide salts with eightfoldcoordinated $\mathrm{CsCl}$ structure in the bulk form instead of the sixfold-coordinated $\mathrm{NaCl}$ (rocksalt) structure. This structure is also met in other caesium halide compounds, except $\mathrm{CsF}$ which experiences a large cation-cation repulsion when eight $\mathrm{Cs}$ cations are packed around the small $\mathrm{F}$ anion [11]. Computational as well as experimental studies have however indicated that small and medium sized $\operatorname{Cs} X$ clusters $(X=\mathrm{Cl}$, $\mathrm{Br}$ or I) have $\mathrm{NaCl}$ structure. It thus follows that at a certain cluster size range a structural phase transition must occur.

Computational studies by Aguado et al. [11,12] involving $(\mathrm{CsCl})_{n} \mathrm{Cs}^{+}$clusters with $n \leqslant 665$ and $(\mathrm{CsI})_{n} \mathrm{Cs}^{+}$with $n \leqslant 14$ have indicated that the ground state structure is a fragment of a $\mathrm{NaCl}$ lattice for small clusters of at least $n \leqslant 50$. For $(\mathrm{CsCl})_{n} \mathrm{Cs}^{+}$clusters it was found that in the four-shell rhombic dodecahedron $(\mathrm{CsCl}$ structure) and the $5 \times 5 \times 7$ cuboid $(\mathrm{NaCl}$ structure), the binding energy per ion values for these two structures with $n=87$ are degenerate. Before this cluster size the $\mathrm{NaCl}$ structure produces larger binding energy per ion values than $\mathrm{CsCl}$ structure and vice versa in larger clusters. Thus one should expect to see more of the clusters with $\mathrm{CsCl}$ structure than $\mathrm{NaCl}$ structure after $n=87$. It was also reported that the polarizability of the ions affects at which cluster size the transition will occur. However, before or after this point, highly symmetric clusters of one isomeric form can be more stable and appear at high abundance, even though the cluster size regime would indicate otherwise. Indeed, stable $(\mathrm{CsI})_{32} \mathrm{Cs}^{+}$and $(\mathrm{CsCl})_{32} \mathrm{Cs}^{+}$clusters with $\mathrm{CsCl}$ structure were experimentally observed in high abundance while near cluster sizes more often favored $\mathrm{NaCl}$ structure [6,7]. Twu et al. [4] concluded that as the mass spectra of $(\mathrm{CsCl})_{n}(n=1-125)$ and $(\mathrm{CsI})_{n}(n=1-100)$ clusters in both cationic and anionic forms were similar to those obtained from other alkali halides with known $\mathrm{NaCl}$ structure, the caesium halides also mostly acquire this structure. Mass spectroscopic investigations for caesium halide clusters much larger than $n \approx 100$ remain very difficult due to the rapidly decreasing detection efficiency for heavy particles.

Besides specific cluster size effects on the transition size, thermal isomerization has been observed for small anionic $\mathrm{Cs} X$ clusters $(X=\mathrm{Cl}, \mathrm{Br}$, or $\mathrm{I})$ using photoelectron 
spectroscopy $[13,14]$. It was reported that at low temperatures cubiclike structures are most abundant while higher temperatures yield also ringlike isomers. This was also studied in a time-resolved manner which revealed a reduced solidlike to liquidlike transition temperature for $\mathrm{CsCl}$ and $\mathrm{CsI}$ clusters.

Temperature is also known to affect bulk alkali halide crystal structure. For example $\mathrm{CsCl}$ is known to have two polymorphs in bulk form. $\alpha-\mathrm{CsCl}$ is stable in room temperature, but when heated to above $460^{\circ} \mathrm{C}$, transformation to $\beta$ - $\mathrm{CsCl}$ with $\mathrm{NaCl}$ structure takes place. While this happens, the lattice expands about $19 \%$ [15]. In clusters such a transition could be possible although the transition temperature would quite likely differ from that of the bulk.

In our study, unsupported neutral clusters of $\mathrm{CsBr}$ were created and studied using synchrotron radiation photoelectron spectroscopy. The investigations were focused on Cs $4 d$ and $\mathrm{Br} 3 d$ core-level regions which were recorded for different mean cluster sizes starting from a few monomers up to several hundreds of atoms per cluster. The cluster response characteristics from the Cs $4 d$ ionized states were compared to modeled ones which indicated a structural phase transition from $\mathrm{NaCl}$ to $\mathrm{CsCl}$ structure. The transition was seen to occur at around 160 atoms per cluster which agrees well with previous computational work [11]. Anomalous features in $\mathrm{Br}$ $3 d$ region cluster response were analyzed using computational methods without reaching complete agreement.

\section{EXPERIMENT}

The experiment was conducted at the I411 undulator beamline [16,17] at the $1.5 \mathrm{GeV}$ MAX II storage ring in MAX-lab (MAX IV Laboratory), in Lund, Sweden. A cluster beam propagating freely in vacuum was intersected at a right angle by a beam of synchrotron radiation. Photon energy was such that photoionization was possible from the selected core orbitals. The emitted photoelectrons were detected by an electrostatic energy analyzer.

Clusters of $\mathrm{CsBr}$ were generated using the EXMEC source [18]. Additional details about the instrument and the alkali halide cluster formation process have been described more thoroughly elsewhere $[10,18]$. Therefore only the experiment specific parameters are mentioned here. Argon was let through a $150 \mu \mathrm{m} \mathrm{LN}_{2}$ cooled convergent-divergent nozzle with a halfopening angle of $10^{\circ}$ into vacuum, creating an Ar cluster beam. After passing through a $300 \mu \mathrm{m}$ skimmer located $12 \mathrm{~mm}$ from the nozzle, the clusters traveled $10 \mathrm{~mm}$ through a heated oven crucible containing the CsBr sample (Sigma Aldrich, 99.95\% purity) where the Ar clusters pick up $\mathrm{CsBr}$ monomers. The number of picked up (adsorbed) particles to the Ar cluster was controlled by adjusting the oven temperature between $425^{\circ} \mathrm{C}$ and $567^{\circ} \mathrm{C}$ with a maximal temperature deviation from the calculated mean of $\pm 2{ }^{\circ} \mathrm{C}$ for a given temperature step. Once inside the Ar cluster, the alkali halide monomers aggregate to form a cluster $[19,20]$. The flight time for clusters from the pick-up oven to the ionization region is of the order $50 \mu \mathrm{s}$. The structure relaxation of the formed $\mathrm{CsBr}$ cluster is expected to happen in the nanosecond timescale.

The nozzle temperature varied between spectra from $123 \mathrm{~K}$ to $132 \mathrm{~K}$ with a mean temperature of $126 \mathrm{~K}$. The maximal temperature deviation from the mean within a given oven temperature step was $\pm 1 \mathrm{~K}$. The Ar stagnation pressure was $1.6 \mathrm{bar} \pm 0.1 \mathrm{bar}$ for the first 7 out of 12 oven temperature steps and 1.5 bar \pm 0.1 bar for the rest.

After the alkali halide oven, the cluster beam intersected the photon beam $\sim 20 \mathrm{~mm}$ from the oven. A photon energy of $133 \mathrm{eV}$ was used with an estimated photon energy resolution of $67 \mathrm{meV}$. The emitted electrons were detected by a Scienta R4000 electron spectrometer set to the "magic" angle of $54.7^{\circ}$ with respect to the horizontally polarized synchrotron radiation. The energy resolution of the electron spectrometer was set to $63 \mathrm{meV}$. The combined photon energy and spectrometer resolution was estimated to be $92 \mathrm{meV}$.

Photoelectron spectra were recorded from Cs $4 d$ and $\mathrm{Br}$ $3 d$ orbitals. Due to the close proximity of binding energies of these levels, the spectra were measured as continuous sweeps between 70-90 eV covering both levels. Energy calibration was performed by recording the photoelectron signal from atomic Ar $3 s$ orbital at $29.239 \mathrm{eV}$ [21].

\section{RESULTS AND DISCUSSION}

The core-level photoelectron spectra are presented in Fig. 1. The data consists of several spectra at different oven temperature which increases from top to bottom. As explained in connection to our earlier measurements [10], this increase in temperature increases alkali halide cluster size. Corresponding alkali metal (panel a) and halide (panel b) spectra are side by side and have relative intensities as shown. Due to the short distance between the alkali halide oven and the photoionization region, some uncondensed vapor is always present in the spectra. Peaks from the uncondensed alkali halide monomers' core ionized states are denoted by $4 d_{5 / 2,3 / 2}^{-1}$ and $3 d_{5 / 2,3 / 2}^{-1}$ for $\mathrm{Cs}$ and $\mathrm{Br}$, respectively. Spectral features associated with the alkali halide clusters have been given either solid $(j=5 / 2)$ or patterned shading $(j=3 / 2)$. Furthermore, only the $j=5 / 2$ components of the doublets are denoted with capital letters $\mathrm{A}-\mathrm{F}$ and are referred to as "peaks A-F" in the subsequent discussion as the $j=3 / 2$ components do not give any additional information about the sites (see Appendix A). The intensities between different datasets were scaled so that the integrated signal from the clusters is the same in each spectrum for easier visual comparison of the cluster response shape. The $\mathrm{CsBr}$ cluster size was estimated based on the cluster aggregation model used earlier to $\mathrm{RbCl}$ and $\mathrm{CsCl}$ clusters [10] with some minor modifications. Most notably, instead of estimating only a single mean alkali halide cluster size, the calculation produces a size distribution which is more appropriate as the experimental spectra are recorded from a distribution of cluster sizes. The mode $N_{\mathrm{m}}$ of the cluster size distribution and the uncertainty of the mode are indicated for each spectrum in Fig. 1. More information about the data treatment (peak fitting) and cluster size estimation process are given in Appendices A and B, respectively.

The most interesting features of the data are seen in the changing cluster related response. Four peaks (A-D) in the Cs region and two in the $\mathrm{Br}$ region experience relative intensity variations as the cluster size increases. Cluster related peaks on the lower binding energy side of the alkali metal $(\mathrm{Rb} 3 d$ and $\mathrm{Cs} 4 d$ ) monomer signal were observed in $\mathrm{RbCl}$ and $\mathrm{CsCl}$ clusters [10] with their behavior as a function of cluster size 

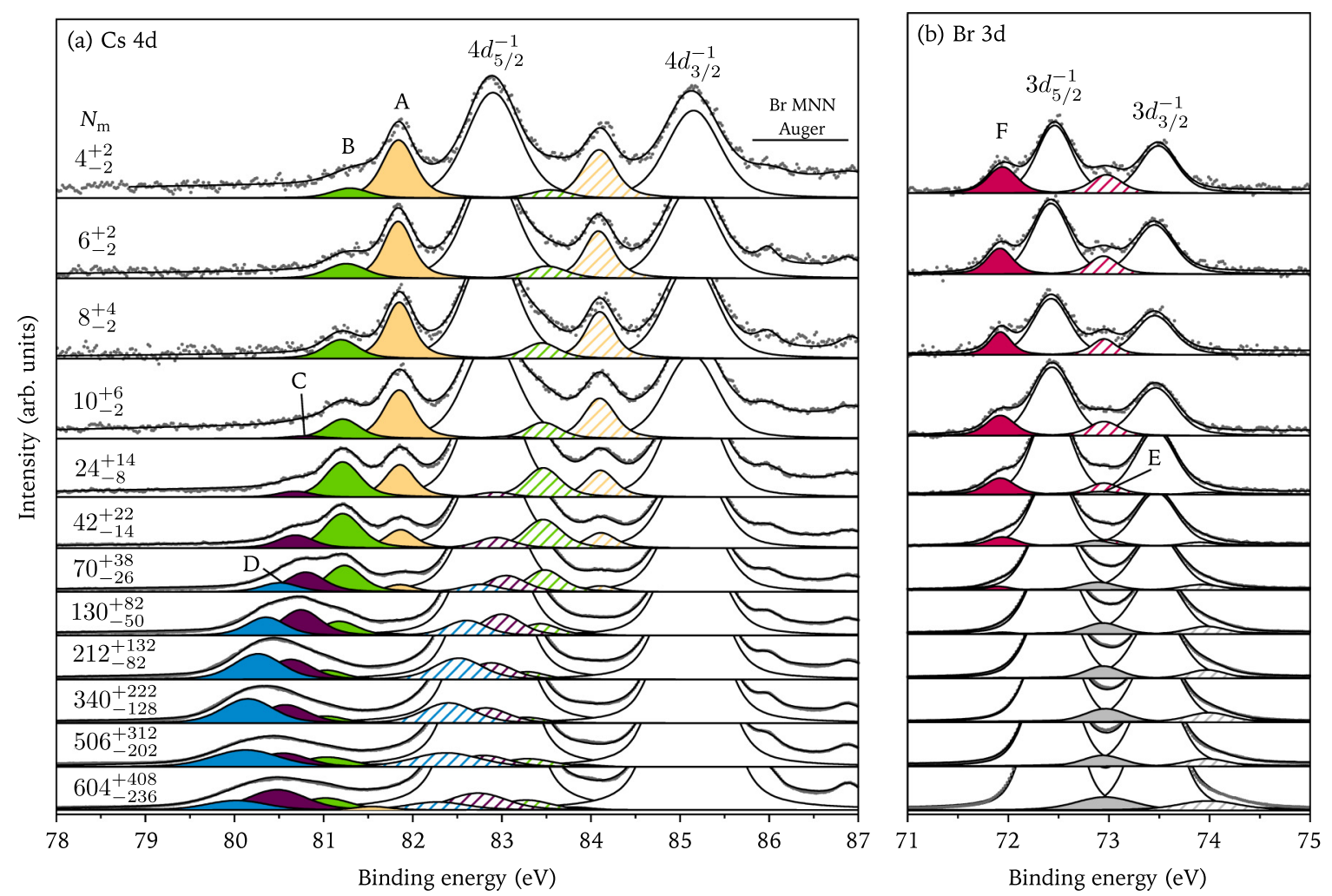

FIG. 1. Photoelectron spectra recorded in (a) Cs $4 d$ and (b) Br $3 d$ regions of CsBr clusters. Peaks related to monomer $\mathrm{Cs} 4 d_{5 / 2,3 / 2}^{-1}$ and $\mathrm{Br}$ $3 d_{5 / 2,3 / 2}^{-1}$ ionized states are denoted in the spectra. In both panels, the $j=5 / 2$ components of the cluster response have been denoted by capital letters A-F and given solid shading while $j=3 / 2$ components have striped pattern fill. Dots denote the experimental data points while the solid thick line is the sum of the individual peak fits. Modeled cluster size distributions are characterized by their mode $N_{\mathrm{m}}$.

qualitatively the same. In the chlorides the halide side $(\mathrm{Cl} 2 p)$ cluster response was only seen on the higher binding energy side of the monomer peaks. In the current set however, part of the $\mathrm{Br} 3 d$ cluster response, denoted as peak $\mathrm{F}$, is clearly seen on the lower binding energy with respect to the monomer signal. The interpretation that peak F originates from the clusters and is not monomer related was verified by comparing the cluster containing spectra to the reference monomer-only spectra. The peak $\mathrm{F}$ decreases in intensity and disappears completely as oven temperature (and mean cluster size) is increased while another, somewhat weaker and broader peak $\mathrm{E}$ rises on the higher binding energy side of the monomer signal. The latter corresponds to the doublet seen in the $\mathrm{Cl} 2 p$ spectra of the alkali chlorides although it is weaker in $\mathrm{CsBr}$.

Peaks A-D correspond to a signal originating from $\mathrm{Cs}^{+}$ ions at sites with increasing coordination [10]. In the $\mathrm{NaCl}$ lattice these are often named corner, edge, face, and bulk sites, respectively. In the $\mathrm{CsCl}$ lattice, the site with lowest coordination is called vertex instead of corner. Peaks A-C can be easily resolved visually up to size 70 . At this point a fourth peak $\mathrm{D}$ has to be fitted in order to explain the low binding energy tail. For cluster size 130, a trimodal spectral structure can be seen, but after this point no clear multimodal structure is apparent. Increasing broadening in each peak increases the peak-to-peak overlap which introduces some uncertainty in the relative weights of the spectral components. This is natural as the number of structural isomers increases rapidly as a function of cluster size. Also for any given coordination site, there is an increasing number of chemical environments where the second and higher coordination shells differ from each other. Although these contribute less to the observed binding energy shifts than the first coordination shell, their net effect is increased peak broadening.

\section{A. Structural phase transition}

The relative area ratios of peaks A-D compared to the total cluster response area are plotted in Fig. 2(a). From hereafter, we refer to them as site fractions. Data points (and corresponding peaks) are denoted with circles (A), squares (B), upward pointing triangles $(\mathrm{C})$, and downward pointing triangles (D) with straight lines connecting them. The uncertainties related to the site fraction values are not trivial to estimate as the error in the fitted area of all the peaks should be taken into account. The Monte Carlo method within the peak fitting program was used to determine the magnitude of error due to random fluctuations in the data, but it was found out that even in dataset with cluster size 8 which has some noise, the error in the overall fitted area was well below $1 \%$. The systematic error of choosing the correct number of peaks for the peak fitting quite likely represents the largest uncertainty in the site fractions when individual peaks can no longer be separated. Based on this qualitative argument, uncertainty in the site fraction values of $\pm 5 \%$ was assigned for all data points smaller than cluster size 130 after which $\pm 10 \%$ was used for all the peaks except peak A. 

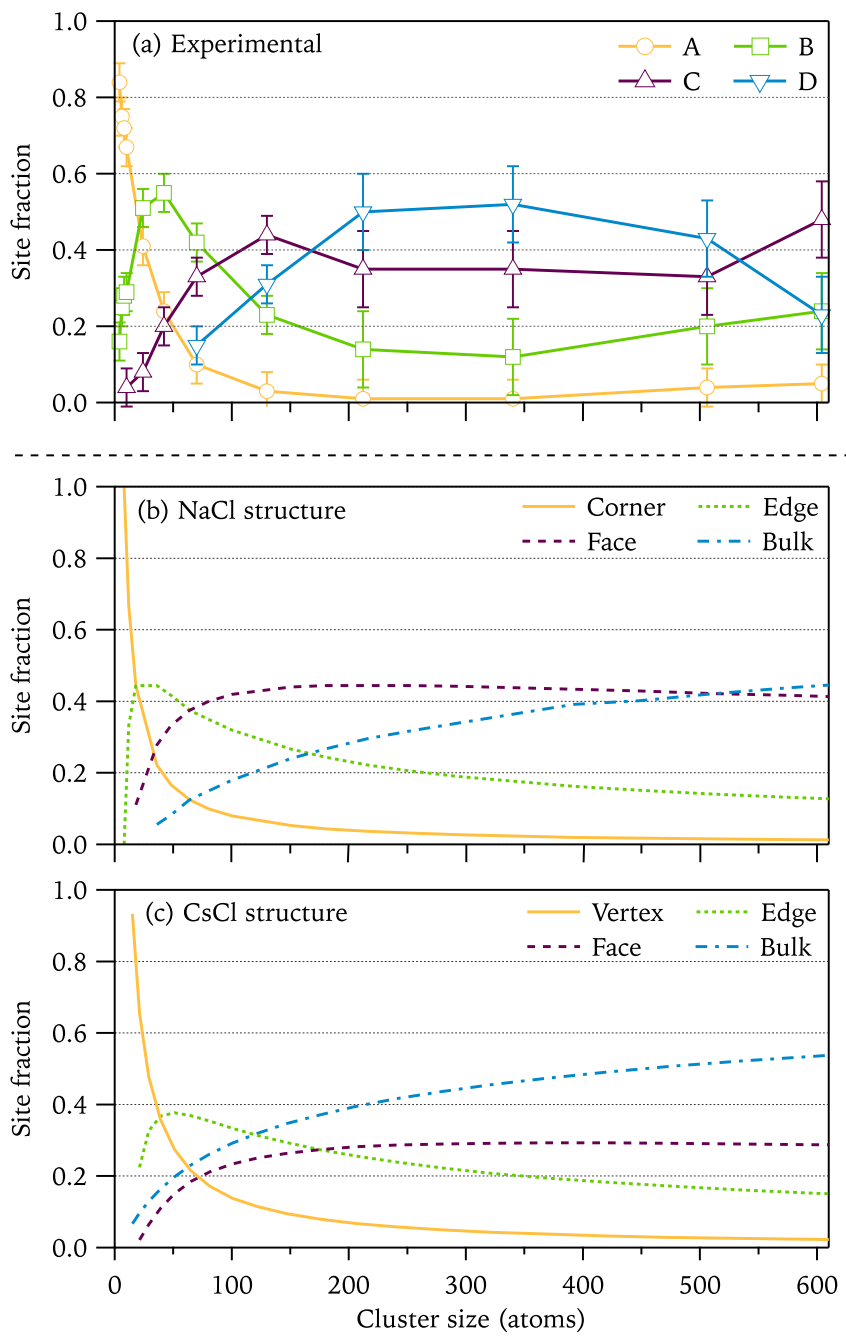

FIG. 2. (a) Experimentally determined relative peak areas, denoted as site fractions, for $\mathrm{CsBr}$. Error bars indicate the estimated uncertainty. The capital letters refer to the peaks observed in the photoelectron spectra. Calculated site fractions for clusters with either (b) $\mathrm{NaCl}$ or (c) $\mathrm{CsCl}$ structure are shown for comparison.

The measured site fractions are accompanied by calculated ones from clusters with $\mathrm{NaCl}$ and $\mathrm{CsCl}$ structures which are shown in Figs. 2(b) and 2(c), respectively. The procedure for calculating the $\mathrm{NaCl}$ structure site fractions follows that given in Ref. [10]. The calculated site fractions in panel (c) represent bcc rhombic dodecahedral clusters which have the same symmetry as bulk $\mathrm{CsCl}$ crystal structure. Data points are calculated only for full atomic shell closings of the structure based on formulas by van Hardeveld and Hartog [22] while intermediate values are interpolated. For $\mathrm{CsCl}$ structure, the coordination of the different sites are 4, 5, 6, and 8 for vertex, edge, face, and bulk, respectively. For $\mathrm{NaCl}$ the numbers are $3,4,5$, and 6 for corner, edge, face, and bulk, respectively.

For both model structures, the least coordinated site (vertex or corner) experiences qualitatively similar monotonous decrease as a function of cluster size. This behavior is also seen in our experimental site fractions for peak A. In both model clusters, the edge site fraction has an initial increase followed by slowly decreasing tail after a maximum at around cluster size 50. The position of the maximum value and the rate of decrease after the maximum are slightly different for the two structures, but overall the behavior is the same. The experimental site fraction curve for peak B qualitatively follows the edge site fraction curves.

$\mathrm{Up}$ to this point the model site fractions for $\mathrm{NaCl}$ and $\mathrm{CsCl}$ structures look similar but for face and bulk sites the behavior becomes very different. While in $\mathrm{CsCl}$ structure the bulk site fraction is larger than the face site fraction, in $\mathrm{NaCl}$ structure the opposite is true up to around 550 atoms per cluster. Comparison to corresponding experimental curves reveals a crossover of curves $C$ and $D$ at $\sim 160$ atoms per cluster. Before this point the experimental curves $\mathrm{C}$ and $\mathrm{D}$ resemble face and bulk curves for $\mathrm{NaCl}$ structure and after that the ordering switches to qualitatively match the $\mathrm{CsCl}$ structure.

As was mentioned in the introduction, calculations reported by Aguado [11] for $(\mathrm{CsCl})_{n} \mathrm{Cs}^{+}$cluster ions place the cluster size at which the cluster binding energy per ion becomes larger in $\mathrm{CsCl}$ structure than the corresponding value in $\mathrm{NaCl}$ structure at $\sim 154$ atoms per cluster if polarization corrections are included $(\sim 216$ when polarization corrections are neglected). As the experimentally observed crossing of the face and bulk site fractions coincides with this size range, it is interpreted as an experimental verification of the structural phase transition.

Starting from the third last data point, the cluster response in photoelectron spectrum experiences a slight deformation where the center-of-mass moves again towards the monomer lines. In principle, this movement can be a combination of peak shifts in energy and relative peak area changes. However, the least-squares fitting suggests that this center-of-mass movement is due to the changing site fractions and not due to binding energy shifts. Similar observations have been made from $\mathrm{RbCl}$ and $\mathrm{CsCl}$ clusters [10], and one possible explanation could be the formation of surface terraces [4] when the cluster size is big enough to host such sites.

We should also note that in the current and previous [10] experiments, the sudden increase in the face, edge, and to some degree the corner site fraction coincides with the almost complete disappearance of the Ar $3 s$ cluster signal. This could indicate that the capacity of the Ar cluster to host additional cluster growth is exhausted and that the evaporative cooling mechanism by the Ar is removed. The decrease of the bulk site fraction could also be due to the inelastic mean free path for the escaping photoelectron being smaller than the cluster size.

\section{B. Anomalous chemical shift in $\mathrm{Br} 3 d$}

Experimental binding energy (chemical) shifts $\Delta E_{\text {exp }}$ between the monomer and cluster peaks have been plotted in Fig. 3(a) as derived from the peak fitting. The uncertainty in the shift magnitude (as indicated by the error bars) was taken to be the Gaussian FWHM after subtracting the beamline and spectrometer broadening contributions from it. The overall trend for all peaks A-D in the alkali metal site is an increasing chemical shift as the cluster size increases. The magnitude of the change in the chemical shift is about $0.5 \mathrm{eV}$ or less within the measured size range.

Unlike the alkali metal levels, the halide side cluster peaks seem to retain their position within the experimental error 

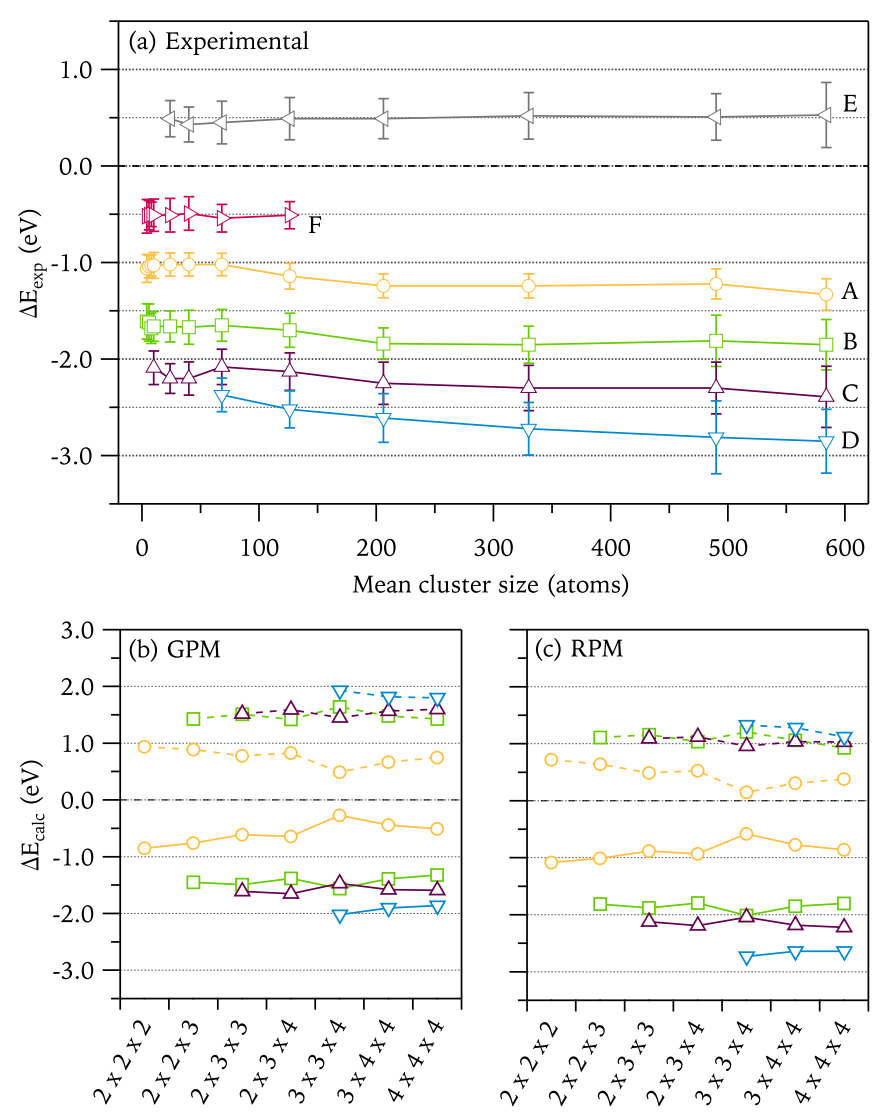

Cluster configuration (i x j x k)

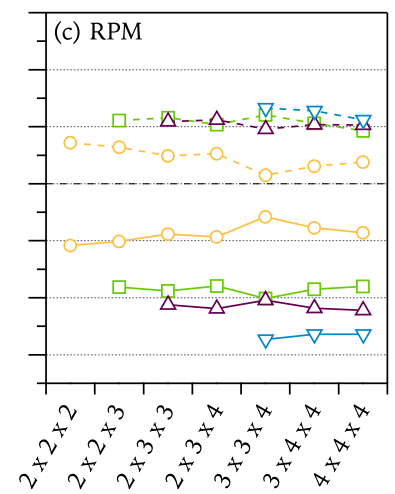

Cluster configuration (i x j x k)

FIG. 3. (a) Experimental binding energy shifts $\Delta E_{\text {exp }}$ between the monomer and cluster peaks A-F. (b) and (c) Calculated binding energy shifts $\Delta E_{\text {calc }}$ for $\mathrm{CsBr}$ clusters as a function of cluster configuration. The results have been computed with (RPM) and without (GPM) the final state relaxation. Markers connected with dashed lines represent shifts for $\mathrm{Br}^{-}$and solid lines for $\mathrm{Cs}^{+}$. Sites are denoted with different markers: Corner (circles), edge (squares), face (upward pointing triangle), bulk (downward pointing triangle).

limits as a function of cluster size. Also, the shift of peak $\mathrm{E}$ in $\mathrm{Br} 3 d$ is similar in magnitude to the $\mathrm{Cl} 2 p$ cluster response reported earlier from $\mathrm{RbCl}$ and $\mathrm{CsCl}$ [10]. The second doublet in $\mathrm{Br} 3 d$, peak $\mathrm{F}$, has a shift of $-0.5 \mathrm{eV}$. As this peak has no counterpart in earlier literature, computational studies were performed to study the possible causes of this observation.

First, chemical shift calculations were performed for small $\mathrm{CsBr}$ clusters with $\leqslant 64$ atoms using a ground state potential model (GPM) and relaxation potential model (RPM) introduced by Davis and Shirley [23-25]. In the former, the shift is taken as the difference in the potential of the reference monomer potential and the potential in the cluster calculated at different sites (corner, edge, etc.). In the RPM, an addition potential term is added to this representing the contribution from the relaxation of the wave functions in the singly ionized state. The electronic wave functions were obtained by using the Orca quantum chemistry program (version 2.9.1) [26]. The calculations were performed in a single configuration Hartree-Fock scheme using the Ahlrichs-VDZ basis sets [27] with effective-core potential for Cs [28]. Further details can be found from Ref. [10]. The cluster geometries were assumed to be simple cuboids where the bond length was taken from optimized structure of $(\mathrm{CsBr})_{4}$ cluster. The reference $\mathrm{CsBr}$ bond length was taken from the experimental value of the monomer [29].

Results from the GPM and RPM calculations have been collected to Figs. 3(b) and 3(c), respectively. The magnitude of the shift is given as a function of cluster configuration. Different configurations are denoted by $i \times j \times k$, where $i, j, k$ are the number of ions along each of the edges of the cuboid cluster having a $\mathrm{NaCl}$ structure. As the results show, $\mathrm{Cs}^{+}$sites acquire only negative shifts while $\mathrm{Br}^{-}$sites have a positive shift. In terms of the different computational models employed, for the $\mathrm{Cs}^{+}$the inclusion of relaxation clearly increases the shift and separation of the shifts from one site to another and does the opposite for the $\mathrm{Br}^{-}$. Comparison of the calculated values for $4 \times 4 \times 4$ clusters with those derived from the spectrum with first appearance of peak doublet $\mathrm{D}$ shows a qualitative agreement for the magnitude of $\mathrm{Cs}^{+}$site shifts but fails to explain the $\mathrm{Br}^{-}$features.

The GPM and RPM calculations involved only pure alkali halide clusters. The cluster formation process involves $\mathrm{Ar}$ clusters as the initial host which are known to be present in the cluster beam in all of the spectra displaying peak F (the Ar $3 s$ cluster signal is seen). Thus it is logical to ask if the Ar cluster could induce a chemical shift to the embedded $\mathrm{CsBr}$. The embedding of the dopant alkali halide cluster species has been reported earlier [20,30], but its effect to the core-level binding energies of the dopant has not been reported. For example, in photoionization of pure Ar clusters the final ionized state is screened by the neighboring Ar atoms via ion induced dipole interaction.

In order to test the notions above, we calculated the chemical shifts of $\mathrm{CsBr}$ induced by surrounding it with 22 $\mathrm{Ar}$ atoms. The number of Ar atoms was chosen such that the computational cost would be reasonable while still including as much of the first coordination shell around the $\mathrm{CsBr}$ monomer as possible. Structures of single $\mathrm{CsBr}$ monomer and $\mathrm{CsBr}$ in $\mathrm{Ar}$ cluster were optimized with the Gaussian program [31] using density functional theory (DFT) with D3 version of Grimme's dispersion with Becke-Johnson damping [32] on def2-TZVP [33,34]/B3LYP [35] level of theory. Br $3 d$ and Cs $4 d$ binding energies were calculated with the Dalton program [36] using the $\Delta$-DFT method $[37,38]$. The calculations were performed using two groups of basis sets: (i) DZP $[39,40]$ for Cs and def2-TZVP for Br and (ii) def2-TZVP basis for both. The latter case involved effective core potentials for $\mathrm{Cs}$, which disabled the possibility to study the $\mathrm{Cs} 4 d$ orbital but improved the $\mathrm{Br}$ results. The B3LYP exchange correlation functional was applied in all cases.

Starting with $\mathrm{CsBr}$ monomer surrounded by $22 \mathrm{Ar}$ atoms, the calculations indicate a chemical shift of $-0.3 \mathrm{eV}$ for $\mathrm{Br}$ $3 d$ and $-0.7 \mathrm{eV}$ for $\mathrm{Cs} 4 d$ with respect to the free monomer values when calculated with the basis group (i). Using group (ii) gives $-0.4 \mathrm{eV}$ for $\mathrm{Br} 3 d$. At least initially it would seem that a number of Ar atoms surrounding the alkali halide could induce a chemical shift to the same direction as observed experimentally. For peak $\mathrm{F}$ the calculations showed that the chemical shift is proportional to the number of Ar atoms as nearest neighbors which was also pointed out by Björneholm et al. [41]. 
Based on the above results it would be tempting to assign the observed anomalous chemical shift to be caused by the presence of Ar. However, since no negative chemical shifts were observed for $\mathrm{Cl} 2 p$ in $\mathrm{CsCl}$ clusters (nor $\mathrm{RbCl}$ clusters) [10], we performed the same calculations for $\mathrm{CsCl}$ monomer surrounded by $22 \mathrm{Ar}$ atoms. With basis group (i), shifts of $-0.3 \mathrm{eV}(\mathrm{Cl} 2 p)$ and $-0.7 \mathrm{eV}(\mathrm{Cs} 4 d)$ are obtained while the use of group (ii) basis set indicated a $-0.4 \mathrm{eV}$ shift for $\mathrm{Cl}$ $2 p$. We can see that the results are essentially the same for the bromide and the chloride so the identity of the halogen atom does not effect the calculated results. This contradiction between the experiment and calculations means that the effect of Ar cannot be assigned as a cause of the anomalous chemical shift.

As the presence of Ar was not found to cause chemical shift uniquely in $\mathrm{Br} 3 d$, we extended the calculations to several other more speculative directions. First we calculated the chemical shift for $\mathrm{CsBr}$ dimer (rhombus geometry) and trimer (ringlike structure). Both gave positive chemical shift for the $\mathrm{Br} 3 d$. Included was also $\mathrm{CsBr}$ monomer optimized with an $\mathrm{H}_{2} \mathrm{O}$ molecule. This was included as it is known that alkali halide samples contain small amounts of water which could, in principle, participate in the cluster formation process. The calculated chemical shift was, however, again positive. In addition, somewhat more exotic candidates $\mathrm{CsBr}_{2}^{-}$superhalogen and $\mathrm{Br}^{-}$anion were ruled out.

As an additional argument for the validity of our observations, we can mention here that a signal with a negative chemical shift in $\mathrm{Br} 3 d$ was also observed for another similar system—small $\mathrm{RbBr}$ clusters (unpublished results). This observation was done in a different experiment using the same cluster source but different oven for heating the alkali halide sample.

\section{CONCLUSIONS}

The main result of the present work is the experimental evidence of the structural phase transition from $\mathrm{NaCl}$ to $\mathrm{CsCl}$ structure in unsupported neutral $\mathrm{CsBr}$ clusters which occurs at around $160_{-60}^{+100}$ atoms per cluster. This size regime is in accord with the theoretical predictions [11] and is supported by the experimental observations of rocksalt structure below the critical size [4] of charged clusters of other caesium halides. It was also demonstrated that important structural information can be obtained even from an ensemble of clusters without size selection. In this case the separation of different coordination sites is largest for alkali halide clusters of less than 100-150 atoms per cluster. In addition, we report an experimental observation of an anomalous negative cluster to monomer chemical shift for one of the cluster related spectra in $\mathrm{Br} 3 d$ region, while most of the studied similar systems indicated positive chemical shift. Modeling and computational studies were performed to explain the observations, but the effects causing the anomalous shift in $\mathrm{Br} 3 d$ region could not be resolved. More extensive experimental studies of the alkali bromide clusters as well as more refined models of the alkali halide clusters are needed in resolving this issue.

\section{ACKNOWLEDGMENTS}

The experiments were performed at MAX-lab (Sweden) at the I411 beamline (proposal number 20130121). EXACTUS doctoral program (Oulun yliopisto) and Jenny ja Antti Wihurin Rahasto are gratefully acknowledged by L.H. for their support. The authors would like to acknowledge the financial support from the Suomen Akatemia. The research leading to these results has received funding from the European Community's Seventh Framework Programme (FP7/2007-2013) CALIPSO under Grant Agreement No. 312284. T.L. acknowledges Vilho, Yrjö, and Kalle Väisälä foundation for their financial support. Computational resources were provided by CSC - IT Center for Science administrated by the Opetus- ja Kulttuuriministeriö. We thank Professor Hans Ågren for sharing his insights about the $\Delta$-DFT method. The authors would also like to thank the MAX-lab staff for their assistance during the experiments.

\section{APPENDIX A: DATA TREATMENT}

All the 12 datasets shown in Fig. 1 were analyzed using a least-squares spectrum fitting program (Igor Pro by Wavemetrics with SPANCF $[42,43]$ package). The fitted peaks all have a symmetric Voigt profile. Beside $\mathrm{CsBr}$ monomer and cluster signal, the $\mathrm{Cs} 4 d$ region contains some overlapping $\mathrm{Br}$ $\mathrm{M}_{4,5} \mathrm{~N}_{2,3} \mathrm{~N}_{2,3}$ Auger lines [44] between 84-88 eV which have been taken into account during the fitting procedure but are not displayed explicitly. Pure monomer spectrum (not shown) was used as a reference for obtaining the Lorentzian full width at half maxima (FWHMs), the spin-orbit splitting, and the relative intensities of the monomer peaks. These were then used as fixed parameters in fitting the cluster response while the Gaussian FWHMs, the absolute peak positions, and absolute intensities were left as free parameters.

\section{APPENDIX B: CLUSTER SIZE ESTIMATION}

Initial Ar cluster size was first estimated based on the cluster source expansion conditions with the Hagena scaling parameter formalism [45] adjusted by Dorchies et al. [46] for $\Gamma^{*}>10^{4}$. The number of picked up $\mathrm{CsBr}$ monomers by the Ar cluster is then iteratively calculated based on the mean free path of the cluster inside the oven having a certain $\mathrm{CsBr}$ vapor pressure [47] determined by the oven temperature. Unlike in our previous calculations [10], the initial Ar cluster size is randomly sampled from a log-normal size distribution with mode given by the Hagena formalism and the FWHM value of the distribution taken to be equal to the mode. The pick-up process is assumed to happen with a sticking and aggregation coefficient of unity, i.e., every monomer encountered by the Ar cluster is adsorbed and aggregates with previously gathered monomers. The modeled $\mathrm{CsBr}$ cluster size distribution is then fitted with a log-normal distribution from which the mode is extracted and presented, e.g., in Fig. 1. Uncertainty in the initial Ar cluster size distribution mode was included by assuming a factor of 2 by which the calculated Ar cluster size mode could vary depending on the specific Hagena model parameters used (see Ref. [48] and references therein). The corresponding $\mathrm{CsBr}$ cluster size distribution modes are then given as the upper and lower limits for the size estimates in Fig. 1. 
The model assumes that the energy released by the initial capture and subsequent coagulation goes to evaporation of $\mathrm{Ar}$ atoms from the host cluster. Sublimation enthalpy of $2.02 \mathrm{eV}$ was used to approximate the energy released by addition of each monomer to the cluster aggregate. As no direct value for this was found in the literature, it was calculated by summing the following reaction chain energies:

$$
\begin{aligned}
\mathrm{CsBr}(\mathrm{g}) & \rightarrow \mathrm{Cs}(\mathrm{g})+\mathrm{Br}(\mathrm{g}) \\
& \rightarrow \mathrm{Cs}^{+}(\mathrm{g})+\mathrm{Br}^{-}(\mathrm{g}) \\
& \rightarrow \mathrm{CsBr}(\mathrm{s}) .
\end{aligned}
$$

The reaction energies are from top to bottom the bond energy $(-4.00 \mathrm{eV})$ [49], the ionization potential of $\mathrm{Cs}(-3.89 \mathrm{eV})$
[50] and electron affinity of $\mathrm{Br}(+3.36 \mathrm{eV})$ [50], and lastly the bulk lattice energy $(+6.55 \mathrm{eV})$ [51]. Calculated values of energy required to evaporate a neutral monomer from $(\mathrm{CsI})_{n} \mathrm{Cs}^{+}$by Aguado et al. [12] varied from $1 \mathrm{eV}$ to $3 \mathrm{eV}$ depending on the cluster size.

If enough alkali halide monomers are captured, it can lead to a complete evaporation of the host Ar cluster [30], and in this case the conversion from one type of cluster to another is complete. In intermediate cases the alkali halide cluster most likely resides inside the Ar cluster forming a core-shell type of structure [30]. During the measurements, the oven temperature was raised and spectra were measured until the alkali halide cluster signal intensity started to decrease. This coincided with the almost complete disappearance of the Ar $3 s$ cluster signal.
[1] S. M. Burrows, O. Ogunro, A. A. Frossard, L. M. Russell, P. J. Rasch, and S. M. Elliott, Atmos. Chem. Phys. 14, 13601 (2014).

[2] L. Miñambres, E. Méndez, M. N. Sánchez, F. Castaño, and F. J. Basterretxea, Atmos. Chem. Phys. 14, 11409 (2014).

[3] K. Mori and K. Hiraoka, Int. J. Mass Spectrom. 269, 95 (2007).

[4] Y. J. Twu, C. W. S. Conover, Y. A. Yang, and L. A. Bloomfield, Phys. Rev. B 42, 5306 (1990).

[5] T. P. Martin, J. Chem. Phys. 81, 4426 (1984).

[6] P. Löffler, Untersuchung der Wachstumssequenzen kleiner Cluster, Ph.D. thesis, Universität Konstanz, 1999.

[7] S. Krückeberg, D. Schooss, M. Maier-Borst, and J. H. Parks, Phys. Rev. Lett. 85, 4494 (2000).

[8] C. Zhang, T. Andersson, S. Svensson, O. Björneholm, M. Huttula, M.-H. Mikkelä, M. Tchaplyguine, and G. Öhrwall, J. Chem. Phys. 134, 124507 (2011).

[9] C. Zhang, T. Andersson, S. Svensson, O. Björneholm, M. Huttula, M.-H. Mikkelä, D. Anin, M. Tchaplyguine, and G. Öhrwall, J. Phys. Chem. A 116, 12104 (2012).

[10] L. Hautala, K. Jänkälä, M.-H. Mikkelä, M. Tchaplyguine, and M. Huttula, Phys. Chem. Chem. Phys. 17, 7012 (2015).

[11] A. Aguado, Phys. Rev. B 62, 13687 (2000).

[12] A. Aguado, A. Ayuela, J. M. López, and J. A. Alonso, Phys. Rev. B 58, 9972 (1998).

[13] F. K. Fatemi, D. J. Fatemi, and L. A. Bloomfield, Phys. Rev. Lett. 77, 4895 (1996).

[14] A. J. Dally and L. A. Bloomfield, Phys. Rev. Lett. 90, 063401 (2003).

[15] S. Chatterji, A. L. Mackay, and J. W. Jeffery, J. Appl. Crystallogr. 4, 175 (1971).

[16] M. Bässler, J.-O. Forsell, O. Björneholm, R. Feifel, M. Jurvansuu, S. Aksela, S. Sundin, S. L. Sorenssen, R. Nyholm, A. Ausmees, and S. Svensson, J. Electron Spectrosc. Relat. Phenom. 101-103, 953 (1999).

[17] M. Bässler, A. Ausmees, M. Jurvansuu, R. Feifel, J.-O. Forsell, P. de Tarso Fonseca, A. Kivimäki, S. Sundin, S. L. Sorensen, R. Nyholm, O. Björneholm, S. Aksela, and S. Svensson, Nucl. Instrum. Methods Phys. Res. 469, 382 (2001).

[18] M. Huttula, M.-H. Mikkelä, M. Tchaplyguine, and O. Björneholm, J. Electron Spectrosc. Relat. Phenom. 181, 145 (2010).

[19] M. Lewerenz, B. Schilling, and J. P. Toennies, J. Chem. Phys. 102, 8191 (1995).
[20] A. Kolmakov, J. O. Löfken, C. Nowak, F. Picucci, M. Riedler, C. Rienecker, A. Wark, M. Wolff, and T. Möller, Eur. Phys. J. D 9, 277 (1999).

[21] A. Kramida, Y. Ralchenko, J. Reader, and NIST ASD Team, NIST Atomic Spectra Database (ver. 5.1) [Online]. Available: http://physics.nist.gov/asd [2014, May 20], National Institute of Standards and Technology, Gaithersburg, MD, (2013).

[22] R. van Hardeveld and F. Hartog, Surf. Sci. 15, 189 (1969).

[23] D. W. Davis and D. A. Shirley, Chem. Phys. Lett. 15, 185 (1972).

[24] D. W. Davis, M. S. Banna, and D. A. Shirley, J. Chem. Phys. 60, 237 (1974).

[25] D. W. Davis and D. A. Shirley, J. Electron Spectrosc. Relat. Phenom. 3, 137 (1974).

[26] F. Neese, Wiley Interdiscip. Rev.: Comput. Mol. Sci. 2, 73 (2012).

[27] A. Schäfer, H. Horn, and R. Ahlrichs, J. Phys. Chem. 97, 2571 (1992).

[28] T. Leininger, A. Nicklass, W. K. Küchle, H. Stoll, M. Dolg, and A. Bergner, Chem. Phys. Lett. 255, 274 (1996).

[29] L. R. Maxwell, S. B. Hendricks, and V. M. Mosley, Phys. Rev. 52, 968 (1937).

[30] A. Kolmakov, J. O. Löfken, C. Nowak, F. Picucci, M. Riedler, C. Rienecker, A. Wark, M. Wolff, and T. Möller, Chem. Phys. Lett. 319, 465 (2000).

[31] M. J. Frisch, G. W. Trucks, H. B. Schlegel, G. E. Scuseria, M. A. Robb, J. R. Cheeseman, G. Scalmani, V. Barone, B. Mennucci, G. A. Petersson, H. Nakatsuji, M. Caricato, X. Li, H. P. Hratchian, A. F. Izmaylov, J. Bloino, G. Zheng, J. L. Sonnenberg, M. Hada, M. Ehara, K. Toyota, R. Fukuda, J. Hasegawa, M. Ishida, T. Nakajima, Y. Honda, O. Kitao, H. Nakai, T. Vreven, J. J. A. Montgomery, J. E. Peralta, F. Ogliaro, M. Bearpark, J. J. Heyd, E. Brothers, K. N. Kudin, V. N. Staroverov, T. Keith, R. Kobayashi, J. Normand, K. Raghavachari, A. Rendell, J. C. Burant, S. S. Iyengar, J. Tomasi, M. Cossi, N. Rega, J. M. Millam, M. Klene, J. E. Knox, J. B. Cross, V. Bakken, C. Adamo, J. Jaramillo, R. Gomperts, R. E. Stratmann, O. Yazyev, A. J. Austin, R. Cammi, C. Pomelli, J. W. Ochterski, R. L. Martin, K. Morokuma, V. G. Zakrzewski, G. A. Voth, P. Salvador, J. J. Dannenberg, S. Dapprich, A. D. Daniels, O. Farkas, J. B. 
Foresman, J. V. Ortiz, J. Cioslowski, and D. J. Fox, Gaussian 09, Revision D.01, Gaussian, Inc., Wallingford, CT, 2013.

[32] S. Grimme, S. Ehrlich, and L. Goerigk, J. Comput. Chem. 32, 1456 (2011).

[33] F. Weigend and R. Ahlrichs, Phys. Chem. Chem. Phys. 7, 3297 (2005).

[34] F. Weigend, Phys. Chem. Chem. Phys. 8, 1057 (2006).

[35] A. D. Becke, J. Chem. Phys. 98, 5648 (1993).

[36] K. Aidas, C. Angeli, K. L. Bak, V. Bakken, R. Bast, L. Boman, O. Christiansen, R. Cimiraglia, S. Coriani, P. Dahle, E. K. Dalskov, U. Ekström, T. Enevoldsen, J. J. Eriksen, P. Ettenhuber, B. Fernández, L. Ferrighi, H. Fliegl, L. Frediani, K. Hald, A. Halkier, C. Hättig, H. Heiberg, T. Helgaker, A. C. Hennum, H. Hettema, E. Hjertenæs, S. Høst, I.-M. Høyvik, M. F. Iozzi, B. Jansík, H. J. A. Jensen, D. Jonsson, P. Jørgensen, J. Kauczor, S. Kirpekar, T. Kjærgaard, W. Klopper, S. Knecht, R. Kobayashi, H. Koch, J. Kongsted, A. Krapp, K. Kristensen, A. Ligabue, O. B. Lutnæs, J. I. Melo, K. V. Mikkelsen, R. H. Myhre, C. Neiss, C. B. Nielsen, P. Norman, J. Olsen, J. M. H. Olsen, A. Osted, M. J. Packer, F. Pawlowski, T. B. Pedersen, P. F. Provasi, S. Reine, Z. Rinkevicius, T. A. Ruden, K. Ruud, V. V. Rybkin, P. Sałek, C. C. M. Samson, A. S. de Merás, T. Saue, S. P. A. Sauer, B. Schimmelpfennig, K. Sneskov, A. H. Steindal, K. O. Sylvester-Hvid, P. R. Taylor, A. M. Teale, E. I. Tellgren, D. P. Tew, A. J. Thorvaldsen, L. Thøgersen, O. Vahtras, M. A. Watson, D. J. D. Wilson, M. Ziolkowski, and H. Ågren, Wiley Interdiscip. Rev.: Comput. Mol. Sci. 4, 269 (2014).
[37] L. Triguero, O. Plashkevych, L. Pettersson, and H. Ågren, J. Electron Spectrosc. Relat. Phenom. 104, 195 (1999).

[38] T. Löytynoja, J. Niskanen, K. Jänkala, O. Vahtras, Z. Rinkevicius, and H. Ågren, J. Phys. Chem. B 118, 13217 (2014).

[39] C. Barros, P. de Oliveira, F. Jorge, A. C. Neto, and M. Campos, Mol. Phys. 108, 1965 (2010).

[40] A. C. Neto and F. Jorge, Chem. Phys. Lett. 582, 158 (2013).

[41] O. Björneholm, F. Federmann, F. Fössing, T. Möller, and P. Stampfli, J. Chem. Phys. 104, 1846 (1996).

[42] E. Kukk, G. Snell, J. D. Bozek, W.-T. Cheng, and N. Berrah, Phys. Rev. A 63, 062702 (2001).

[43] E. Kukk, K. Ueda, U. Hergenhahn, X.-J. Liu, G. Prümper, H. Yoshida, Y. Tamenori, C. Makochekanwa, T. Tanaka, M. Kitajima, and H. Tanaka, Phys. Rev. Lett. 95, 133001 (2005).

[44] Z. Hu, A. Caló, J. Nikkinen, T. Mattila, E. Kukk, and H. Aksela, J. Chem. Phys. 121, 8246 (2004).

[45] O. F. Hagena, Surf. Sci. 106, 101 (1981).

[46] F. Dorchies, F. Blasco, T. Caillaud, J. Stevefelt, C. Stenz, A. S. Boldarev, and V. A. Gasilov, Phys. Rev. A 68, 023201 (2003).

[47] G. E. Cogin and G. E. Kimball, J. Chem. Phys. 16, 1035 (1948).

[48] O. Björneholm, F. Federmann, F. Fössing, and T. Möller, Phys. Rev. Lett. 74, 3017 (1995).

[49] E. K. Parks and S. Wexler, J. Phys. Chem. 88, 4492 (1984).

[50] CRC Handbook of Chemistry and Physics, 96th ed., edited by W. M. Haynes (Taylor \& Francis Group, Boca Raton, FL, 20152016).

[51] D. Cubicciotti, J. Chem. Phys. 33, 1579 (1960). 